\title{
A Case of Hemiplegia Vegetativa Alterna, Paroxysmal Sympathetic Hyperactivity and Ogilvie's Syndrome: The Role of Central Sympathetic Pathways in Their Pathophysiology
}

\author{
Peter Y. M. Woo ${ }^{\mathrm{a}, ~ b}$, Joanna W. K. Ho ${ }^{\mathrm{a}}$, Sharon T. M. Cheng ${ }^{\mathrm{a}}$, Kar-Ming Leung ${ }^{\mathrm{a}}$, Kwong-Yau Chan ${ }^{\mathrm{a}}$
}

\begin{abstract}
We describe a patient with aneurysmal subarachnoid hemorrhage who developed hemiplegia vegetativa alterna (HVA), a rare brainstem syndrome characterized by contralateral hemiplegia, hemisensory loss, hemihyperhidrosis and ipsilateral Horner's syndrome. The patient also experienced paroxysmal sympathetic hyperactivity (PSH) and colonic pseudo-obstruction, known as Ogilvie's syndrome. A magnetic resonance imaging (MRI) scan revealed a focal lesion at the anterolateral mesencephalon. It is believed that lesions of this region can interrupt central sympathetic pathways and account for this unique combination of syndromes. This first documented case provides an opportunity to review the relevant neuroanatomy and contributes to cumulative clinical evidence verifying the excitatory-inhibitory ratio (EIR) disconnection theory for PSH.
\end{abstract}

Keywords: Hemiplegia vegetativa alterna; Paroxysmal sympathetic hyperactivity; Ogilvie's syndrome; Sympathetic dysautonomia

\section{Introduction}

Hemiplegia vegetativa alterna (HVA) is the clinical syndrome of contralateral hemiparesis, hemisensory loss, hemihyperhidrosis and ipsilateral Horner's syndrome $[1,2]$. The term vegetativa alterna denotes that a single brainstem lesion manifests with ipsilateral and contralateral, i.e. crossed, signs of autonomic ("vegetative") sympathetic nervous system dysfunction. Fewer than five cases have been reported and most were a result of stroke involving the occlusion of posterior cerebral artery (PCA) perforators that supply the anterolateral mesencephalon [1-3].

We describe a patient who suffered from aneurysmal sub-

Manuscript accepted for publication July 30, 2014

aDepartment of Neurosurgery, Kwong Wah Hospital, Hong Kong, SAR, China ${ }^{b}$ Corresponding Author: Peter Y. M. Woo, Department of Neurosurgery, Kwong Wah Hospital, Hong Kong, SAR, China.

Email: peterymwoo@gmail.com

doi: http://dx.doi.org/10.14740/jnr284w arachnoid hemorrhage and exhibited HVA as a consequence of mesencephalic injury. The patient also experienced paroxysmal sympathetic hyperactivity (PSH) and recurrent colonic pseudo-obstruction, known as Ogilvie's syndrome. PSH is identified as a pathological state of elevated sympathetic activity characterized by episodic tachycardia, hypertension, tachypnea, hyperthermia diaphoresis and decerebrate dystonia occurring in $10 \%$ of severe traumatic brain injury patients [4]. Although the pathogenesis has yet to be elucidated the excitatory-inhibitory ratio (EIR) disconnection theory postulates that certain brainstem centers are inhibitory in nature and when severely injured, the spinal cord is released from higher regulation leading to sympathetic hyperactivity [5]. Colonic pseudo-obstruction is a gastro-intestinal emergency with brain injury accounting for $10 \%$ of cases $[6,7]$. It is understood that sympathetic hyperactivity also plays an important role in its pathophysiology [7].

To our knowledge, this is the first documented case of HVA, PSH and colonic pseudo-obstruction in the literature. We propose that the three conditions are related to an interruption of central sympathetic pathways and present clinicoradiological evidence to demonstrate that focal lesions of the anterolateral mesencephalon can account for this unique presentation.

\section{Case Report}

A 46-year-old male experienced generalized seizures with a Glasgow coma score of 7/15. A computed tomography (CT) brain scan revealed Fisher grade three diffuse subarachnoid hemorrhage and catheter angiography depicted a ruptured 2.5 $\times 2.5 \mathrm{~mm}$ (height $\times$ width) right ophthalmic segment internal carotid artery aneurysm. Stent-assisted coil embolisation was performed 2 days later with successful aneurysm obliteration. Post-operatively the patient developed intracranial hypertension and left hemiplegia. A CT angiogram showed right middle cerebral artery (MCA) vasospasm with no evidence of carotid dissection. Hyperosmolar therapy and propofol sedation was therefore initiated for intracranial pressure control. Two days later the patient developed medically refractory intracranial hypertension and a repeat $\mathrm{CT}$ scan revealed a massive right MCA region infarction exerting mass effect on the right cerebral peduncle. A decompressive craniectomy and right anterior

Articles $\left(\right.$ The authors | Journal compilation @ J Neurol Res and Elmer Press Inc ${ }^{\mathrm{TM}}$ | www.neurores.org

This is an open-access article distributed under the terms of the Creative Commons Attribution License, which permits unrestricted use, distribution, and reproduction

in any medium, provided the original work is properly cited 

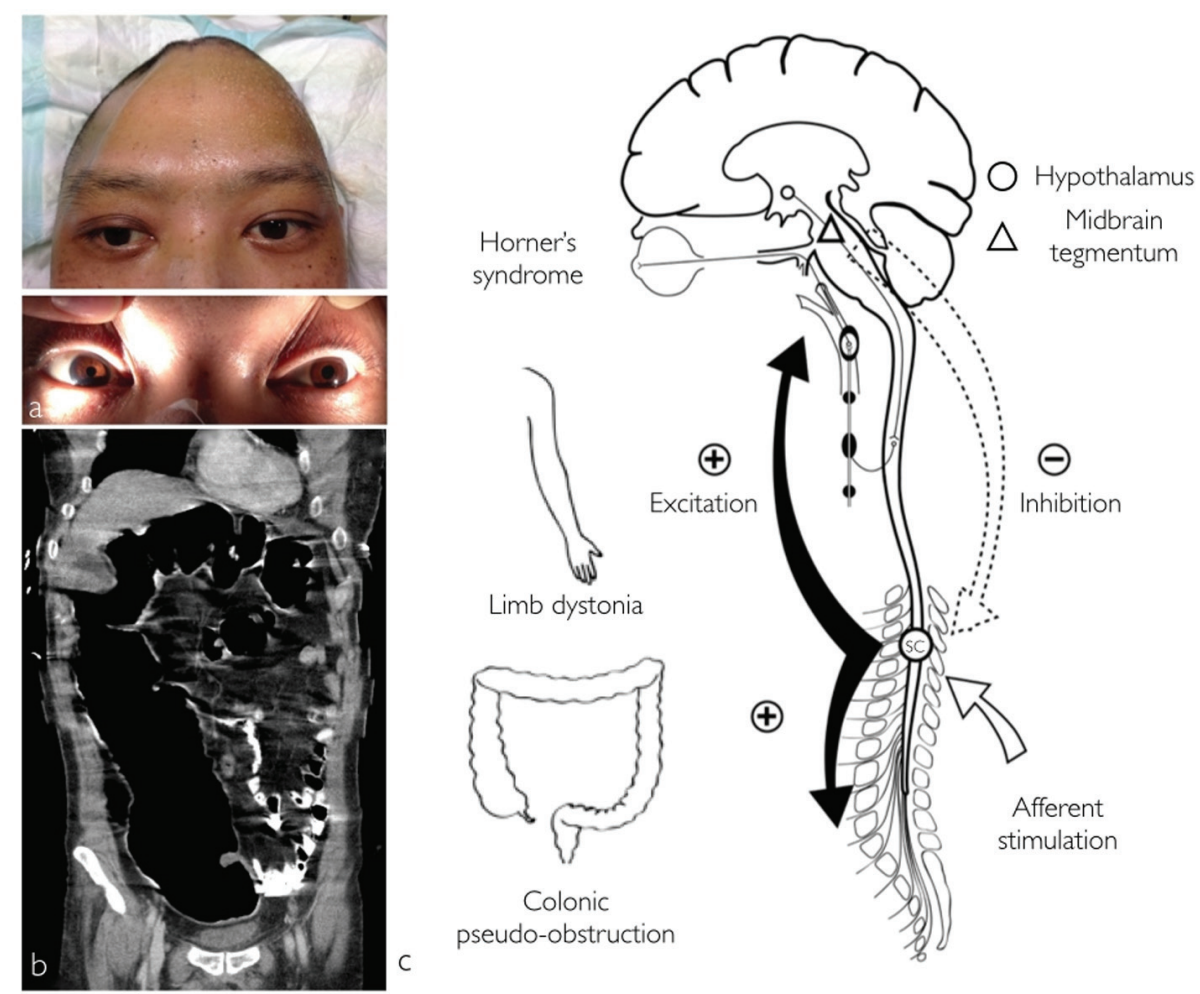

Figure 1. Photographs displaying right side Horner's syndrome with right hemifacial anhidrosis and left hemihyperhidrosis. Partial eyelid ptosis and meiosis was also observed (a). Acute colonic pseudo-obstruction (b) coronal abdominal computed tomography. Schematic illustrating the pathophysiology behind the concomitant occurrence of central Horner's syndrome, limb dystonia, colonic pseudo-obstruction and PSH (c). A mesencephalic lesion causes disruption of the hypothalamospinal sympathetic pathway produces Horner's syndrome and according to the EIR model, loss of normal tonic inhibition of spinal centers from the brainstem (dotted-arrow). Due to this disinhibition minor afferent stimulation (white arrow) elicits spinal allodynia and diffuse spinal cord excitation (black arrow) with PSH (SC, spinal cord center).

temporal lobectomy was performed.

Post-operatively the patient developed episodic decerebrate limb dystonia. Each episode was accompanied with tachycardia of 130 - 160 beats per minute and hypertension ranging from $160 / 120$ to $220 / 140 \mathrm{~mm} \mathrm{Hg}$ (mean arterial pressure was $196 \mathrm{~mm} \mathrm{Hg}$ ). There was also tachypnea of $30-40$ breaths per minute, hyperthermia of up to $41^{\circ} \mathrm{C}$ and profuse diaphoresis limited to the left side of the body and left face. The paroxysms were self-limiting occurring 6 - 8 times per day and lasted for a mean duration of $40 \mathrm{~min}$. Due to their sudden intermittent nature and associated decerebrate limb dystonia, seizures were initially suspected, but were ruled out by electroencephalography. Investigations also excluded an underlying infection. It became evident that they were triggered by physical stimulation such as chest physiotherapy, bed-turning and bathing.

In addition, right side Horner's syndrome was diagnosed when ipsilateral facial anhidrosis, made even more apparent during these diaphoretic episodes, right pupillary miosis and partial eyelid ptosis (Fig. 1a) were observed. The patient also experienced recurrent abdominal distension due to colonic pseudo-obstruction that lasted a month despite normal serum electrolyte levels and medications impairing intestinal motility such as opioids were stopped (Fig. 1b). The hypersympathetic paroxysms eventually resolved after 3 weeks and the colonic pseudo-obstruction responded to regular intravenous neostigmine and rectal tube insertion. Magnetic resonance imaging (MRI) performed 6 weeks after the initial insult showed evidence of right anterolateral mesencephalic injury, whereas the hypothalamus and the remaining brainstem were intact (Fig. 2). Upon discharge the patient was able to obey commands with left hemiplegia, hemisensory loss and right Horner's syndrome.

\section{Discussion}

Our case not only offers an opportunity to review central sympathetic pathway neuroanatomy, but also supports recent pathophysiological theories for sympathetic hyperactivity observed in patients with brainstem injury.

\section{HVA: clinical evidence of central antagonistic sympathetic pathways}

Central Horner's syndrome is a consequence of first-order neuron disruption of the hypothalamospinal sympathetic tract, 

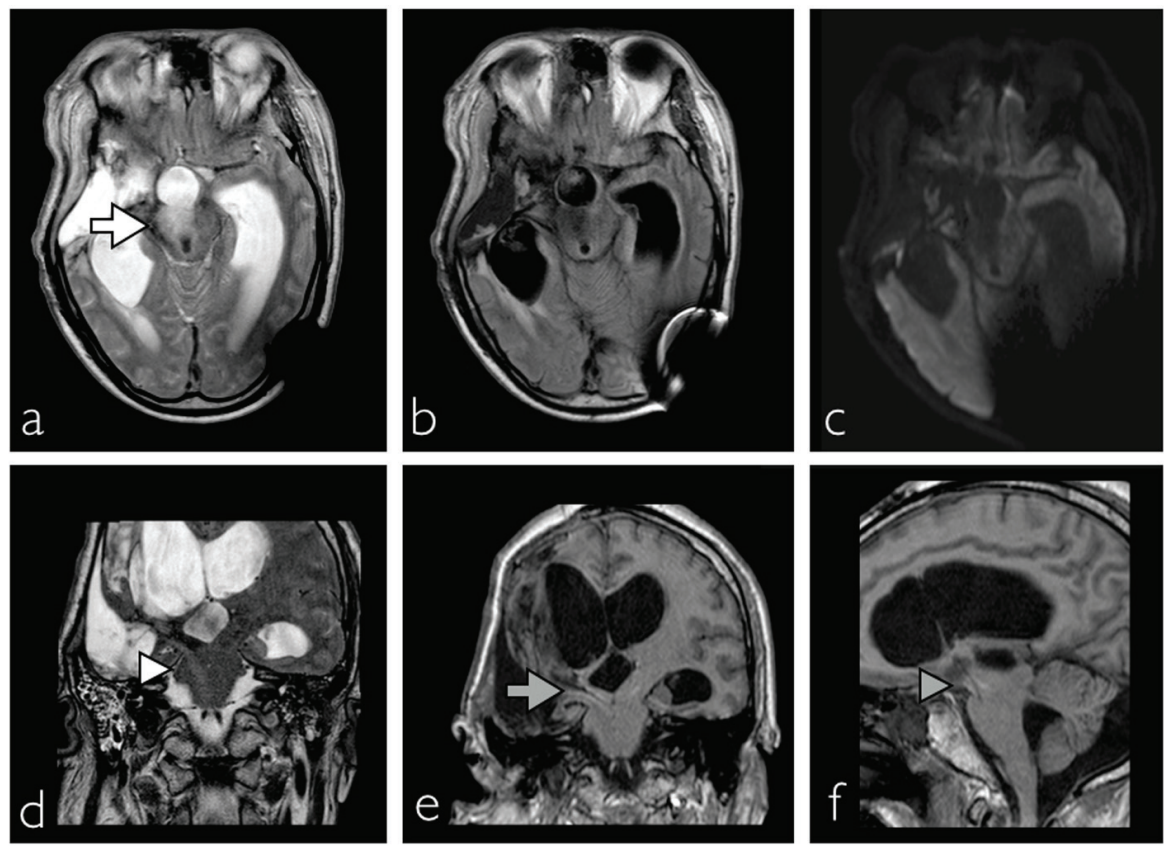

Figure 2. MRI showing evidence of right cerebral peduncle and lateral tegmental injury of the mesencephalon ((a) white arrow, axial T2 sequence; (b) fluid attenuated inversion recovery FLAIR sequence; (c) diffusion-weighted imaging). Injury as a result of downward transtentorial herniation leading to injury of the hypothalamospinal sympathetic tract ((d) white arrowhead, coronal T2; (e) gray arrow, coronal FLAIR; (f) gray arrowhead, sagittal, FLAIR).

also known as the oculosympathetic tract. Most cases are associated with lateral medullary stroke or less frequently cervical syringomyelia or trauma [8]. Although the classical three-neuron pathway is well recognized and the literature is redolent of reports depicting peripheral pre- and post-ganglionic lesions, anatomic descriptions of the central pathway have not received as much attention. The central efferent pathway projects uninterrupted from the posterolateral hypothalamus (the oculosympathetic center of Karplus and Kreidl), to the ciliospinal center of Budge at the intermediolateral horn of the T1 to T2 spinal cord levels [9]. At the mesencephalon crossing of inhibitory sympathetic fibers occur at the ventral tegmental decussation of Forel. The remaining excitatory fibers descend uncrossed within the lateral funiculus of the brainstem tegmentum $[9,10]$. Contralateral hemihyperhidrosis has been reported in several patients with cortical, thalamic and brainstem lesions and is attributed to an interruption of a putative decussating sympatho-inhibitory perspiratory pathway [11-14]. The additional presence of ipsilateral central Horner's syndrome in HVA, reflecting concomitant sympatho-excitatory pathway disruption, suggests that these antagonistic sympathetic tracts are in close proximity at the mesencephalic tegmentum.

For our case it is postulated that transtentorial herniation caused proximal PCA compression against the free edge of the tentorium that compromised perfusion of its peduncular and circumferential perforators. The ensuing focal infarction of the anterolateral mesencephalon involving the corticospinal, spinothalamic and sympathetic tracts offers an appropriate explanation for the patient's clinical presentation. This hypothesis is supported by previous descriptions of HVA patients with mesencephalic stroke due to proximal PCA occlusion [1-3]. We presume that the medially located oculomotor and trochlear nuclei were spared in our patient as they receive separate perfusion from the paramedian mesencephalic branches of the basilar artery [9].

\section{PSH and Ogilvie's syndrome}

PSH has been identified in more than 350 patients with various brain insults, most commonly trauma $[4,15]$. The consequence of this condition is cerebral hypermetabolism leading to secondary brain injury and intracranial hypertension $[4,15,16]$. Although several diagnostic criteria for PSH have been proposed, no consensus driven set has been recognized [4].

In addition to the well-recognized signs of PSH, we theorize that colonic pseudo-obstruction can be another manifestation of this syndrome. The condition, typified by massive colonic dilatation without mechanical blockage, is thought to be a result of increased sympathetic drive during times of extreme physiological stress [7]. This hypersympathetic state is believed to stimulate colonic smooth muscle inhibitory neurons that regulate colonic peristalsis (Fig. 1c) [7]. A vicious cycle of continued colonic dilatation is established when distension activates mural mechanoreceptors of the colo-colonic reflex to further suppress motility [7]. This theory is supported by the accepted therapeutic effectiveness of neostigmine, a parasympathomimetic cholinesterase inhibitor, and guanethidine, a sympathetic antagonist [6]. Most respond well to conservative management and single dose intravenous neostigmine, but our patient's recurrent episodes of pseudo-obstruction may be 
ascribed to PSH.

\section{The EIR model for PSH}

The presence of PSH in our patient also implies a possible greater role of descending central sympatho-inhibitory pathways beyond sudomotor regulation. Disconnection theories causing loss of autonomic regulation currently predominate discussions on PSH pathophysiology $[4,5]$. Conventional models have focused on higher cortical or subcortical lesions leading to disinhibition of the hypothalamus or brainstem, the postulated main drivers for the hypersympathetic state $[4,5]$. However, there are a number of reservations regarding this proposition. First, the sympatho-excitatory center model implies that the hypothalamospinal pathways must remain intact, but autopsy and MRI studies of PSH patients with diffuse axonal injury exhibit extensive mesencephalic injury with normal hypothalami $[5,16]$. Secondly, decerebrate posturing during paroxysms suggests a focal brainstem lesion and verifies the results of a case series describing an association between dysautonomia, muscle rigidity and mesencephalic compression [16]. Finally, conventional disconnection theories fail to account for paroxysm triggering by external afferent stimulation, a hallmark of PSH $[4,16]$.

An alternative disconnection theory, the EIR model, advocates that central autonomic centers are conversely inhibitory in nature and sympathetic hyperactivity is released from the spinal cord when these centers are damaged (Fig. 1c) [5]. This concept provides a simpler rationale that does not require the integrity of efferent pathways and offers an explanation for the triggering phenomenon. The model is based on the premise that sympathetic activity depends on the balance between afferent stimulation at the spinal cord level and modulation from brainstem centers especially at the mesencephalic tegmentum $[5,16]$. At the dorsal horn of the spinal cord tonic descending sympathetic inhibition normally prevents allodynia, which refers to the misinterpretation of non-noxious stimuli as nociceptive [5]. It is proposed that brainstem sympatho-inhibitory pathway injury causes spinal allodynia with the resultant excitatory drive establishing connections between afferent and sympathetic pathways through the entire length of spinal cord [5]. An analogous example is autonomic dysreflexia in high thoracic spinal cord injury where symptoms are elicited by minor stimuli such as muscle stretching and pain $[5,16]$.

\section{Conclusion}

We present a patient with a unique combination of syndromes produced by a lesion located at the anterolateral mesencephalic tegmentum. It is proposed that disruption of the central sympathetic pathways at this location can account for the spectrum of autonomic disturbances observed. This case highlights the possible role of descending sympatho-inhibitory pathways in PSH pathophysiology although it is acknowledged that clinical evidence is limited and investigatory tools to ratify this hypothesis have yet to be developed.

\section{References}

1. Bassetti C, Staikov IN. Hemiplegia vegetativa alterna (ipsilateral Horner's syndrome and contralateral hemihyperhidrosis) following proximal posterior cerebral artery occlusion. Stroke. 1995;26(4):702-704.

2. Uca AU, Kozak HH. Could hemiplegia vegetativa alterna be a cerebral sign of heart calve disease? Archives of neuropsychiatry. 2014;51:95-96.

3. Rossetti AO, Reichhart MD, Bogousslavsky J. Central Horner's syndrome with contralateral ataxic hemiparesis: a diencephalic alternate syndrome. Neurology. 2003;61(3):334-338.

4. Perkes I, Baguley IJ, Nott MT, Menon DK. A review of paroxysmal sympathetic hyperactivity after acquired brain injury. Ann Neurol. 2010;68(2):126-135.

5. Baguley IJ. The excitatory:inhibitory ratio model (EIR model): An integrative explanation of acute autonomic overactivity syndromes. Med Hypotheses. 2008;70(1):2635.

6. Ponec RJ, Saunders MD, Kimmey MB. Neostigmine for the treatment of acute colonic pseudo-obstruction. N Engl J Med. 1999;341(3):137-141.

7. Saunders MD. Acute colonic pseudo-obstruction. Gastrointest Endosc Clin N Am. 2007;17(2):341-360, vi-vii.

8. Walton KA, Buono LM. Horner syndrome. Curr Opin Ophthalmol. 2003;14(6):357-363.

9. Kardon R. Anatomy and physiology of the autonomic system. In: Miller NR, Newman NJ, eds. Walsh and Hoyt's clinical neuro-ophthalmology. Vol 1. 6 ed. Maryland: Lippincott Williams \& Wilkins; 2005:649-714.

10. Marx JJ, Iannetti GD, Mika-Gruettner A, Thoemke F, Fitzek S, Vucurevic G, Urban PP, et al. Topodiagnostic investigations on the sympathoexcitatory brain stem pathway using a new method of three dimensional brain stem mapping. J Neurol Neurosurg Psychiatry. 2004;75(2):250255.

11. Kim BS, Kim YI, Lee KS. Contralateral hyperhidrosis after cerebral infarction. Clinicoanatomic correlations in five cases. Stroke. 1995;26(5):896-899.

12. Awada A, Ammar A, al-Rajeh S, Borollosi M. Excessive sweating: an uncommon sign of basilar artery occlusion. J Neurol Neurosurg Psychiatry. 1991;54(3):277-278.

13. Sato K, Nitta E. [Pontine hemorrhage presenting with Foville syndrome and transient contralateral hyperhidrosis]. Rinsho Shinkeigaku. 2000;40(3):271-273.

14. Rey A, Marti-Vilalta JL, Abellan MT. [Contralateral hyperhidrosis secondary to the pontine infarct]. Rev Neurol. 1996;24(128):459-460.

15. Fernandez-Ortega JF, Prieto-Palomino MA, Garcia-Caballero M, Galeas-Lopez JL, Quesada-Garcia G, Baguley IJ. Paroxysmal sympathetic hyperactivity after traumatic brain injury: clinical and prognostic implications. J Neurotrauma. 2012;29(7):1364-1370.

16. Baguley IJ, Nicholls JL, Felmingham KL, Crooks J, Gurka JA, Wade LD. Dysautonomia after traumatic brain injury: a forgotten syndrome? J Neurol Neurosurg Psychiatry. 1999;67(1):39-43. 